\title{
ILCEA
}

Revue de l'Institut des langues et cultures

d'Europe, Amérique, Afrique, Asie et Australie

18 | 2013

Les frontières dans le monde hispanique

\section{Expressions et perception de la frontière de Grenade dans l'œuvre historiographique d'Alfonso de Palencia}

Expressions and Perception of the Frontier of Granada in Alfonso de Palencia's Historiographic Opus

Expresiones y percepción de la frontera de Granada en la obra historiográfica de Alfonso de Palencia

\section{Alice Carette}

\section{OpenEdition}

\section{Journals}

Édition électronique

URL : http://journals.openedition.org/ilcea/2033

DOI : 10.4000/ilcea.2033

ISSN : 2101-0609

Éditeur

UGA Éditions/Université Grenoble Alpes

Édition imprimée

ISBN : 978-2-84310-251-6

ISSN : 1639-6073

Référence électronique

Alice Carette, «Expressions et perception de la frontière de Grenade dans l'œuvre historiographique d'Alfonso de Palencia », ILCEA [En ligne], 18 | 2013, mis en ligne le 11 juillet 2013, consulté le 02 mai 2019. URL : http://journals.openedition.org/ilcea/2033 ; DOI : 10.4000/ilcea.2033

Ce document a été généré automatiquement le 2 mai 2019.

(C) ILCEA 


\title{
Expressions et perception de la frontière de Grenade dans l'œuvre historiographique d'Alfonso de Palencia
}

\author{
Expressions and Perception of the Frontier of Granada in Alfonso de Palencia's \\ Historiographic Opus \\ Expresiones y percepción de la frontera de Granada en la obra historiográfica de \\ Alfonso de Palencia
}

Alice Carette

1 La question de la frontière dans l'Espagne médiévale est intimement liée à la Reconquista, nom donné par l'historiographie ultérieure à cet affrontement séculaire ${ }^{1}$, qui s'achève, à proprement parler, à l'issue de la série de campagnes victorieuses du roi Ferdinand III de Castille et de León (prises de Cordoue en 1236, de Jaén en 1246 et de Séville en 1248). Ces conquêtes donnent naissance à une nouvelle entité régionale qui est repeuplée et intégrée à la couronne de Castille : l'Andalousie chrétienne, dont la société est vouée à la guerre contre les habitants musulmans du petit royaume de Grenade. Ce territoire est généralement appelé par les historiens des $\mathrm{xx}^{\mathrm{e}}$ et $\mathrm{xxI}^{\mathrm{e}}$ siècles la "frontière de Grenade ", même si le référent spatial de cette expression contemporaine, comme celui de ses équivalents médiévaux, déborde parfois la seule Andalousie chrétienne².

Le royaume de Grenade (dont le roi devient le vassal du roi de Castille dès 1246) est l'ultime vestige de l'ancienne Espagne musulmane. À partir de la victoire chrétienne du Salado (1340), il est définitivement isolé militairement, ne recevant plus d'appui de l'autre rive de la Méditerranée. Hormis quelques expéditions chrétiennes ou musulmanes en terre ennemie, les relations entre les deux États sont très longtemps dominées par une situation de paix armée régie par un système de trêves; ces dernières sont renouvelées régulièrement moyennant le paiement d'un lourd tribut par le roi de Grenade ${ }^{3}$. Ce n'est qu'en 1482 que commence la phase active et finale de la conquête du royaume; elle 
s'achèvera en janvier 1492, avec l'entrée dans la ville de Grenade d'Isabelle de Castille et Ferdinand d'Aragon ${ }^{4}$.

3 Avant d'aborder la question de l'expression de la frontière de Grenade dans l'œuvre d'Alfonso de Palencia, un point théorique préalable sur le concept de frontière et son expression dans l'Espagne médiévale est nécessaire. Je proposerai donc ici une rapide synthèse sur ces questions, traitées plus amplement dans ma thèse ${ }^{5}$.

Parmi les très nombreux travaux historiques consacrés au concept de frontière dans l'Espagne médiévale et à son évolution au fil des siècles, l'un des plus éclairants et synthétiques est l'article de Miguel Ángel Ladero Quesada intitulé « Sobre la evolución de las fronteras medievales ${ }^{6} »$. L'académicien y situe au XIII ${ }^{e}$ siècle le moment où le concept de frontière prend son sens moderne, c'est-à-dire celui de "limite entre deux États ", selon la définition du Trésor de la langue française. Il écrit ainsi :

A mediados del siglo XIII tocaba a su fin el tiempo histórico en el que los países de la España cristiana habian tenido como objetivo político principal la guerra y las conquistas territoriales frente a al-Andalus. Terminaba entonces la época de la Frontera por excelencia, de las sucesivas marcas fronterizas establecidas frente al islam andalusí desde finales del siglo XI para ser, en algún momento, sobrepasadas. La última Frontera, con mayúscula, fue la de Granada, que sólo concluirá con la conquista de finales del siglo XV aunque después conservó vigencia en el orden administrativo interno de la Corona de Castilla.

Pero, al mismo tiempo, se habían precisado cada vez mejor las fronteras - esta vez con minúscula-, límites o «rayas» entre los reinos de la España cristiana a lo largo del proceso de diferenciación política, paralelo al de expansión territorial, que ocurrió entre los siglos XI y XIII, tanto en las tierras ya dominadas por los poderes cristianos desde épocas anteriores como en las que se iban incorporando a sus respectivos ámbitos. [...]

Todos [los historiadores] coinciden en afirmar que desde mediados o finales del siglo XIII se inició y consolidó la tendencia a precisar mejor las fronteras como manifestación de los límites a los que llegaba el poder de un rey, que comenzaba a encarnar la noción de Estado y la soberanía aunque ambas no estuvieran aún precisadas con la claridad que se alcanzó en tiempos posteriores ${ }^{7}$.

5 Ladero Quesada s'appuie notamment sur les travaux de José Antonio Maravall qui affirmait que l'on ne peut pas appliquer au haut Moyen Âge le concept de frontière tel qu'on l'entend aujourd'hui, mais plutôt celui de $\mathrm{Marca}^{8}$. Maravall définissait, par ailleurs, ce concept dans les termes suivants :

La Marca es un concepto eminentemente militar que, de su propia naturaleza, hace alusión a una zona inestable, imprecisa, sin organización fija, sin establecimiento de un señorío seguro y administrativamente canalizado, lugar de correrías y de cambios bruscos, donde no dominan más que los que alli permanecen de hecho, y aun éstos tan sólo sobre la tierra que pisan. Responde a los procedimientos bélicos de los primeros siglos medievales y aun al sentido entero de la vida, acosado siempre periféricamente por unos límites agresivos. Se ha dicho muchas veces que marca significa frontera y, por extensión, región fronteriza; pero se ha tenido siempre la tendencia a considerar esas fronteras en un sentido moderno, como una línea trazada con precisión, hasta la que llega, uniformemente por todos sus puntos, la acción de un poder interior. [...] La marca o frontera de la alta Edad Media es una región de nadie -despoblada, dicen con frecuencia las Crónicas y diplomas [...] ${ }^{9}$.

6 Pour Maravall comme pour Ladero Quesada, deux concepts de frontière se succèdent donc dans l'Espagne médiévale. D'abord, celui de Marca-frontera, que l'on appellera "frontière médiévale ", et que Ladero appelle la «Frontière par excellence " (avec, comme il le précise, une majuscule $)^{10}$. Cette forme de frontière, qui n'est pas une ligne mais une "zone ", une bande de territoire, est dépourvue de dimension administrative ; elle correspond, par ailleurs, à ce qu'André Bazzana appelle "frontière de reconquête " 
ou « frontière en marche ${ }^{11}$. Ensuite, celui de frontera-raya, qui s'applique, à partir du XIII ${ }^{\mathrm{e}}$ siècle, aux limites entre États chrétiens (dont Ladero Quesada précise qu'elle s'écrit avec une minuscule) et que l'on appellera « frontière moderne ».

7 Pour Ladero Quesada, la frontière de Grenade, contemporaine des premières « frontières modernes » péninsulaires appartient encore, cependant, à la première catégorie. Elle est, pour lui la dernière frontière médiévale, alors que, pour André Bazzana, elle se situe plutôt de côté moderne du concept $\mathrm{t}^{12}$. Pour ma part, je considère la frontière de Grenade comme un moyen terme entre "frontière médiévale » et "frontière moderne », dans la mesure où elle emprunte des caractéristiques à ces deux états du concept. Elle est, en effet, un territoire, mais un territoire bordé d'une ligne militarisée; elle est à la fois provisoire et pérenne, vouée à disparaître et institutionnalisée par les liens de vassalité qui unissent le roi de Grenade à celui de Castille, ainsi que par les divisions administratives des deux États ; elle est, enfin, théoriquement « en marche » mais, de fait, quasi-immobile pendant plus de deux siècles.

8 L'évolution du fait frontalier au cours du Moyen Âge est indissociable de l'évolution de son expression ${ }^{13}$. Pour le haut Moyen Âge, comme on l'a vu, Maravall préfère parler de Marca. Le mot latin médiéval frontaria et sa traduction castillane frontera (dérivés du latin frontis, frontis, front d'une armée) apparaissent, quant à eux, au XI ${ }^{\mathrm{e}}$ siècle. Selon Philippe Sénac ${ }^{14}$ et Pascal Burési ${ }^{15}$, ils sont employés, dès le départ, dans des contextes relatifs à la lutte contre les musulmans. J'ai montré, dans ma thèse, comment leur emploi évolue et se généralise dans les chroniques castillanes, au cours $d u$ xiII ${ }^{e}$ siècle, venant se substituer progressivement à tous les termes latins et castillans utilisés jusque-là pour désigner les limites matérielles ou mentales (politiques, militaires, religieuses, identitaires) entre deux territoires ${ }^{16}$.

9 En raison du statut intermédiaire et paradoxal de la frontière de Grenade, à la fois médiévale et moderne, son expression lexico-syntaxique et toponymique dans les chroniques en castillan des $\mathrm{XIV}^{\mathrm{e}}$ et $\mathrm{XV}^{\mathrm{e}}$ siècles est spécifique et complexe ${ }^{17}$. Du point de vue lexico-syntaxique, d'abord, elle se distingue de celle des frontières entre la Castille et les royaumes chrétiens péninsulaires: en effet, alors que les textes mentionnent «la frontera de Aragón » ou « la frontera de Portugal », par exemple, ils utilisent, pour désigner la frontière de Grenade l'expression «la frontera de los moros ». Cette asymétrie souligne la différence de perception entre, d'un côté, des États nommés par leur nom, avec lesquels la Castille possède des frontières politiques, et, de l'autre, celui de Grenade, avec lequel la séparation est d'ordre religieux plus que politique. L'expression de la frontière de Grenade est spécifique, ensuite, du point de vue toponymique. L'ensemble territorial mitoyen du royaume de Grenade, constitué des royaumes de Séville, Cordoue et Jaén (et doté de structures sociales, économiques et politiques spécifiques) est désigné dans les textes rédigés en castillan par deux termes qui, dans une certaine mesure, semblent entrer en concurrence: l'un est Andalucía, l'autre la Frontera qui, précédé d'un article défini et sans complément, devient un véritable toponyme ${ }^{18}$. Néanmoins, si tous deux fonctionnent bien souvent comme des synonymes, ils ne sont pas systématiquement interchangeables. Le toponyme Andalucía, qui correspond à une réalité administrative et juridique, est strictement réservé à la désignation de l'Andalousie chrétienne. Il est employé principalement dans les passages consacrés aux événements internes à cette dernière, dans des contextes de paix avec Grenade, qui coïncident, le plus souvent, avec de fortes tensions au sein de la société chrétienne andalouse. La Frontera, qui ne renvoie en revanche à aucune division administrative, est une expression à connotation 
nettement militaire : c'est pourquoi elle est majoritairement présente dans les récits de périodes de guerre ouverte entre la Castille et Grenade pour désigner aussi bien l'Andalousie chrétienne, base arrière des combats, que le royaume de Grenade, terrain des affrontements ${ }^{19}$ ou la guerre elle-même ${ }^{20}$. À la dualité conceptuelle de la frontière de Grenade, à la fois médiévale et moderne, correspond donc, dans la langue castillane, une dualité toponymique.

$\mathrm{Au}$ sein d'une historiographie royale castillane par ailleurs relativement homogène en termes d'idéologie, de structures et d'expression linguistique, Alfonso de Palencia, précurseur de l'humanisme en Espagne, occupe une place singulière pour plusieurs raisons qu'il ne convient pas de détailler ici $^{21}$. Arrêtons-nous simplement sur la plus évidente, qui est d'ordre linguistique: Palencia, formé à l'humanisme italien, rédige les quatre Décades des Gesta Hispaniensia et la Guerra de Granada (Bellum adversus Granatenses) en latin, contrairement à ses pairs ${ }^{22}$. Or, le latin humaniste, distinct du latin médiéval et qui, selon l'expression de Carmen Codoñer, « réinvente » le latin classique ${ }^{23}$, n'offre pas le même lexique que ce dernier ni d'équivalents stricts des mots castillans pour décrire le monde. En raison du choix de sa langue d'expression, Palencia ne dispose donc pas des mêmes outils que ses contemporains pour nommer ce qui l'entoure. C'est pourquoi, pour nommer la région frontalière entre la Castille et le royaume de Grenade, il n'utilise pas de traduction ou de transposition du syntagme castillan la Frontera ni du toponyme Andalucía , mais un lexique, une syntaxe et une toponymie qui lui sont propres. Nous verrons que ces spécificités sont le fruit à la fois de la culture humaniste de l'auteur et de son fort parti pris idéologique.

11 Avant d'examiner et d'analyser l'expression de la frontière de Grenade dans l'œuvre d'Alfonso de Palencia, nous montrerons en premier lieu comment il désigne les frontières entre la Castille et ses autres voisins. Nous verrons ensuite comment et pourquoi l'auteur, en adaptant de manière personnelle la toponymie classique de l'Espagne méridionale, transmet de la frontière de Grenade une image aussi complexe que fluctuante. Enfin, nous analyserons la nature et la fonction du discours géographique de Palencia sur le royaume de Grenade.

\section{Limites, confins, contiguïté : l'expression des frontières chez Alfonso de Palencia}

Pour appréhender la singularité de la dénomination de la frontière de Grenade dans l'œuvre de Palencia, il convient d'y examiner les expressions des limites et de la contiguïté entre l'ensemble des États péninsulaires, sachant que le mot latin médiéval frontaria n'existe pas dans le lexique de l'auteur.

\subsection{Les frontières entre la Castille et les États chrétiens péninsulaires}

Le substantif latin le plus souvent employé par Palencia pour exprimer les notions que recouvre le concept de frontière est fines (pluriel de finis), qui signifie en latin la « limite d'un territoire » et que l'on rencontre dans l'historiographie castillane médiévale en latin avant l'apparition de frontaria ${ }^{24}$. On trouve ainsi à plusieurs reprises, dans les Gesta Hispaniensia et Bellum adversus Granatenses, les expressions Cantabriae fines, pour désigner 
la frontière avec la Navarre, fines Aragoniae ou fines Portugaliae $e^{25}$. Plus rarement, l'auteur utilise le substantif finitima, utilisé dans le même sens que fines. Il écrit ainsi, en relatant les circonstances de la constitution de la Hermandad General, entre 1465 et 1467, que les habitants de la région de Ségovie, suivis de ceux d'autres régions du royaume de Castille créent de nouvelles Hermandades, inspirées des anciennes, « a finibus Aragoniae usque ad finitima Lusitania ${ }^{26}$ ».

Comme le mot frontera, fines et finitima renvoient soit à la périphérie d'un territoire (par rapport à un centre), soit à la limite de ce territoire avec un autre, cette limite peut être interne ou externe. Palencia explique ainsi que Carlos de Viana s'est allié avec le noble castillan Pedro Fernández de Velasco, afin de compter un allié « in illis Cantabriae fines » : il se réfère ici à la frontière extérieure de la Navarre, côté castillan, le comté de Haro étant situé en Castille ${ }^{27}$. Un peu plus loin, il explique comment les troupes françaises - dont le roi, Louis XI, s'est allié à Jean II d'Aragon pour l'aider à réprimer le soulèvement catalan - entrent en Aragon " per finitima Cantabriae ", c'est-à-dire par la frontière interne de la Navarre. Il s'agit encore de frontière interne quand Palencia écrit comment, en 1453, le roi de Navarre entre, depuis l'Aragon « dans les frontières de la Castille » (« ex Aragonia [...] fines Castellae ingreditur $»)^{28}$.

15 Fines, suivi d'un génitif, se rapporte donc tantôt à la limite interne, tantôt à la limite externe d'un territoire ou d'un État. Pour dissiper cette ambiguïté, Palencia utilise parfois un double génitif. Afin de situer la ville de Logroño, il écrit ainsi : «Lucronem, quae ditionis Castellae finibus Cantabriae contigua est ${ }^{29}$. Comme dans cet exemple, l'auteur a souvent recours à plusieurs termes, pour situer un lieu, là où le castillan utilise simplement le syntagme adjectival frontero de ou le syntagme prépositionnel en la frontera de suivi du nom d'un État. Il peut en effet utiliser un adjectif (contiguus/a, proximus/a, finitimus/ $a^{30}$ ) ou un verbe (adiacet). Ainsi, pour situer la ville de Molina (actuelle Molina de Aragón), il précise : «Molina in Tagitana provincia adiacet finibus Aragonia ${ }^{31}$ ".

Certes, le latin de Palencia ne dispose pas d'un mot aussi polysémique que le castillan frontera pour exprimer à la fois l'extrémité d'un État, sa contiguïté avec un autre et la fonction militaire de cette zone; mais la limite entre deux États, dans les exemples examinés, est très nette. Un lieu est situé d'un côté ou de l'autre de la frontière, une action ou un déplacement effectués à partir d'un État vers un autre. Une telle précision reflète indéniablement la reconnaissance et, déjà, la stabilité des frontières politiques avec les autres États chrétiens, même en cas de conflit armé. L'expression de la frontière entre le royaume de Grenade et les territoires de l'Andalousie chrétienne ou de la région de Murcie est plus complexe et problématique.

\subsection{Lexique et syntaxe de la frontière de Grenade}

Tentons à présent de déterminer les principaux traits des expressions de la frontière entre la Castille et Grenade, nombreuses surtout dans la première Décade et Bellum adversus Granatenses, textes quirelatent les années les plus intenses d'activité militaire contre Grenade.

\section{La frontière de Grenade côté musulman}

18 Pour les zones frontalières entre la Castille et les royaumes chrétiens, on l'a vu, Palencia utilise le plus souvent fines, suivi du nom du royaume chrétien au génitif. Pour désigner 
les zones frontalières avec le royaume de Grenade, il n'emploie pas le génitif du nom du royaume (Granatae) mais celui de l'adjectif qui désigne ses habitants, Granatenses. Ainsi, à propos de la forteresse de Jiquena, il écrit, dans la première Décade " $\operatorname{ar}[x]$ Xiquene in provincia Carthaginensi adiacen $[s]$ Granatensium finibus ${ }^{32}$ ». On retrouve la construction fines Granatensium dans Bellum lorsque Palencia écrit, par exemple, qu'un Catalan nommé Juan de Cardona arriva à Cordoue, en 1487, « antequam rex Fernandus exercitum in Granatensium fines educeret ${ }^{33}$ ». Un autre terme, confinium, est également employé dans le récit pour évoquer les gouverneurs militaires de forteresses frontalières, situées, écrit Palencia, « in confinio Granatensium ${ }^{34} »$. Pour désigner les territoires grenadins voisins de ceux des chrétiens, Palencia le Castillan utilise donc des constructions qui mettent en avant le peuple du royaume de Grenade, et non la dimension politique du royaume nasride. Il se rapproche en cela des chroniqueurs d'expression castillane, qui préfèrent parler de la frontera de los Moros plutôt que de la frontera de Granada, formule consacrée par les historiens contemporains mais quasiment absente des chroniques des $\mathrm{XIV}^{\mathrm{e}}$ et XV$^{\mathrm{e}}$ siècles $^{35}$. Il est vrai que l'on ne trouve à aucun moment, ni dans les Décades, ni dans Bellum, l'expression fines Maurorum, qui serait l'équivalent de la formulation castillane ; toutefois, dans bien des cas, les termes Granatenses et Mauri sont interchangeables ou complémentaires ${ }^{36}$. Pour l'auteur, comme pour ses contemporains, la démarcation entre la Castille et Grenade n'est donc pas une frontière politique entre deux États, mais une frontière religieuse entre deux populations de confessions distinctes. Or l'islam, dont la présence de fait est constatée, n'est pas reconnu comme légitime propriétaire de la région qu'il occupe.

Désigner la frontière interne du royaume de Grenade, c'est aussi désigner tout son territoire : celui-ci est en effet, dans son ensemble, une frontière, d'une part en tant que théâtre et objet de l'affrontement avec la Castille, d'autre part en tant que région intermédiaire entre l'Europe chrétienne et les rives musulmanes de la Méditerranée.

Dans la première Décade, le toponyme Granata n'apparaît que très rarement, le plus souvent pour désigner la cité de Grenade elle-même. Ainsi, lorsque pour évoquer la ville d'Alcalá la Real, il la qualifie de "urb[s] munitissim[a] finitim[a]que Granatae ${ }^{37}$ », c'est sa proximité avec la capitale de l'État nasride qu'il souligne. Les emplois du toponyme Granata, seul ou associé à un autre terme, pour désigner le royaume musulman sont beaucoup plus rares. Dans la première Décade, il est rare que le toponyme serve à désigner sans ambiguïté ${ }^{38}$ le royaume de Grenade dans son ensemble ; c'est néanmoins le cas dans l'expression Granatae regnum, sur laquelle nous reviendrons ${ }^{39}$. Si ces deux syntagmes expriment et donc reconnaissent, l'existence d'une entité politique grenadine, le territoire est envisagé, le plus souvent, du simple point de vue de son étendue, par des expressions qui désignent ses terres agricoles, du type " campos granatenses", " campos Granatensium $»^{40}$ ou « hortos Maurorum ${ }^{41} »$.

21 La désignation du royaume de Grenade évolue nettement dans Bellum adversus Granatenses, œuvre consacrée à la guerre finale menée par les Rois catholiques. Si l'on trouve, comme dans les Décades, les expressions « campos granatenses ${ }^{42}$ », « campos Granatensium », " agro[s] Granatensium $»^{43}$ ou "terras Granatensium ${ }^{44}$ ", dénuées de toute connotation politique, l'adjectif granatensis, au singulier ou au génitif pluriel, est désormais associé à d'autres substantifs, comme provincia. L'auteur se réfère ainsi à plusieurs reprises à la « provincia granatensis" ou à la «provincia Granatensium »45. À défaut d'être reconnu par ses frontières, destinées à être abolies, le royaume de Grenade apparaît donc au moins comme une entité douée d'une existence administrative. Par ailleurs, contrairement à ce 
qu'il faisait dans les Décades, Palencia, dans Bellum adversus Granatenses, utilise à de nombreuses reprises des termes qui renvoient à une entité politique, comme ditio ${ }^{46}$ (forme archaïque de Dicio, onis, qui signifie puissance, empire, domination, autorité ${ }^{47}$ ) ou regnum $^{48}$. L'utilisation de regnum va de pair avec celui de rex, très fréquemment associé lui aussi, dans Bellum adversus Granatenses, au génitif du toponyme Granata ou à celui de l'adjectif substantivé Granatenses ${ }^{49}$. La présence de ces expressions est d'autant plus fréquente dans le texte que, au cours du récit, plusieurs rois de Grenade, en conflit les uns avec les autres, se succèdent, voire cohabitent ${ }^{50}$.

Au fil du récit de la guerre de Grenade, on trouve donc de plus en plus d'expressions lexicales du pouvoir politique nasride. La présence de ce type d'expression traduit indéniablement un intérêt pour le pouvoir politique grenadin, interlocuteur inévitable, et dont les divisions servent les intérêts des chrétiens engagés dans des négociations avec Boabdil. De manière logique, en revanche, on ne trouve aucune expression de type fines Granatae ou finitima Granatae: si les frontières du royaume de Grenade n'ont jamais été désignées clairement parce qu'elles n'étaient pas reconnues par les chrétiens, elles ont encore moins de raisons de l'être dans le récit de la guerre de Grenade, qui relate le démantèlement progressif de l'État nasride. Dès le début de la guerre, la frontière relativement stable qui - même si elle n'était pas officiellement reconnue - existait de fait depuis 1246 recommence à avancer au détriment des musulmans; elle redevient alors une "frontière de reconquête ", une frontière " en marche ", lieu de colonisation et de repeuplement ${ }^{51}$.

\section{Le versant chrétien de la frontière de Grenade}

De même, on trouve des constructions adjectivales pour situer des territoires grenadins par rapport à leur proximité avec les territoires chrétiens, comme la plaine fertile (vega) de Grenade, qualifiée de "planicie contermina fere finibus nostris ${ }^{52}$ ». Outre les différentes constructions syntaxiques autour du substantif fines, on trouve des constructions adjectivales (avec adiacentis, finitima) pour indiquer la situation frontalière de certains lieux de l'Andalousie chrétienne avec Grenade : la ville de Jaén est ainsi définie comme voisine des Grenadins (" [ingreditur] Giennensem urbem finitimam Granatensibus $\left.{ }^{53} »\right)$. Un autre secteur chrétien frontalier de Grenade, le royaume de Murcie (à l'est de Grenade), est également désigné par des périphrases contenant l'adjectif finitimum. À propos d'Alfonso Fajardo, grand noble murcien, Palencia écrit ainsi :

Faiardo, qui Carthaginem novam et loca omnia finitima Mauris litori Mediterranei sinus adiacentibus possidebat (nam de Beticis mentio facta est) ${ }^{54}$.

24 Aux adjectifs qui expriment la proximité, l'auteur en ajoute parfois d'autres lorsqu'il souhaite signaler le caractère militarisé d'une place frontalière : il qualifie ainsi Alcalá la Real de ville très armée et voisine de Grenade ( Alcalam Regiam urbem munitissimam finitimamque Granatae $\left.e^{55} »\right)$. Il fait également des allusions régulières aux installations militaires chrétiennes, comme après le récit de la prise de Jimena sous Henri IV, dont il écrit : « reliquos [...] fines Beticae Granatensibus propinquiores presidiis quoque firmari jubet ${ }^{56}$ ».

Comme l'illustre ce dernier exemple, les substantifs ou adjectifs qui désignent le versant chrétien de la frontière de Grenade sont, par ailleurs, souvent accompagnés de toponymes, pour préciser à quel secteur de l'Andalousie chrétienne on se rapporte. Si l'on trouve, par exemple, des expressions du type "Cordobensium fines ${ }^{57}$ ", fines est aussi 
parfois associé à l'adjectif beticus ${ }^{58}$ ou au génitif du toponyme Betica ( fines Betica $\left.e^{59} »\right)$. L'utilisation complexe de ce dernier toponymemérite un examen approfondi.

\section{Vandalia et Betica : la toponymie palentinienne de la frontière de Grenade}

Le latin de Palencia, comme on l'a vu, ne possède pas d'équivalent du toponyme castillan la Frontera, qui désigne à la fois les régions castillanes voisines du royaume de Grenade, la ligne fortifiée qui les en protège et, dans certains cas, le royaume de Grenade lui-même. L'auteur utilise, cependant, deux toponymes polyréférentiels, qui peuvent servir, selon le cas, à désigner l'Andalousie chrétienne, le royaume de Grenade ou les deux espaces à la fois. L'un est Betica, du nom de la province de l'Hispanie romaine qui a perduré, de même que les autres divisions administratives romaines, sous les Wisigoths. L'autre est Vandalia, adaptation castillane de l'arabe Al-Andalus (nom donné par les musulmans à la totalité de la Péninsule ibérique et dont le nom des Vandales a longtemps été considéré, à tort, comme l'origine étymologique $\left.{ }^{60}\right)$.

\subsection{Le toponyme Betica et ses référents spatiaux}

Le nom de Betica a longtemps été employé par l'historiographie castillane en latin, par imitation, depuis Hydace et Isidore de Séville : en premier lieu pour désigner l'ancienne province romaine, maisaussi, dans les chapitres consacrés à l'après 711 , les territoires musulmans de la péninsule. On le trouvait pour la dernière fois dans le Chronicon Mundi de Lucas de Tuy (1230-1239) ; un autre texte contemporain, la Crónica latina de los Reyes de Castilla (1226-1230), l'avait déjà remplacé, pour nommer les territoires musulmans, par Handalucia $^{61}$. Chez Palencia, qui le réintroduit dans l'historiographie pour des raisons complexes ${ }^{62}$, ses référents spatiaux sont également divers.

\section{Betica comme dénomination de l'Andalousie chrétienne}

Chez Palencia, Betica désigne d'abord le territoire chrétien composé des juridictions de Séville, Cordoue et Jaén, celui que les chroniques en castillan nomment Andalucía, par un jeu d'appropriation partiel et complexe du toponyme arabe Al-Andalus ${ }^{63}$. Cette relation apparaitt très clairement lorsque, dans la première Décade, le parcours de la cour castillane est ainsi retracé :

Idque peragunt curiales per Beticam a Gienensi urbe Cordubam, e Corduba Astinam vel Astigiema, inde Carmonem, postea Hispalim progressi [...] ${ }^{64}$.

Betica, pour désigner l'Andalousie chrétienne, peut alors être utilisé seul, comme ici : " Dum rex Beticam pervagaretur, quotidianis nunciis solicitabatur a marchione [...]65.» Plus souvent, Betica est associé à provincia. Dans la deuxième Décade, Palencia qualifie les troubles ourdis en Andalousie par don Juan Pacheco de "futura damna quae provinciae Betice moliebatur Magister ${ }^{66} »$. Le mot provincia exprime la connotation administrative et officielle attribuée par Palencia à Betica, dimension confirmée notamment par la traduction du titre d'Adelantado de Andalucía (que le castillan appelle aussi Adelantado de la Frontera), par «preses Beticae ${ }^{67}$ ». De même que Betica désigne l'Andalousie chrétienne, de même l'adjectif substantivé Betici, précédé ou non d'un possessif, désigne ses habitants ( nostri Betici », «Beticos nostros ", " vires omnium Beticorum » $\left.{ }^{68}\right)$. Betica est enfin associée à ses 
centres urbains, avec des substantifs comme civitas (" civitatum Beticae provinciae $\left.{ }^{69} »\right)$ ou populus (« [Fernandus Rex] [...] qui Hispalim, Cordubam multosque alios Betice populos e manibus Maurorum eripuit $\left.{ }^{70} »\right)$.

Comme le toponyme Andalucía dans les textes en castillan, Betica est donc utilisé pour désigner l'Andalousie chrétienne, notamment dans sa dimension administrative. Mais cette dernière n'est pas l'unique référent spatial du toponyme romain.

\subsection{Une Bétique aux contours variables}

Dans les passages cités précédemment, Betica ne désigne qu'une partie de l'ancienne Bétique romaine, plus étendue que l'Andalousie chrétienne du $\mathrm{xv}^{\mathrm{e}}$ siècle. Or, si Betica désigne fréquemment l'Andalousie chrétienne, le toponyme peut avoir également, sous la plume de l'auteur, un référent spatial plus étendu.

Dans son Universal Vocabulario en latín y en romance (1490), Palencia délimite la Bétique dans son extension est-ouest, et précise qu'elle tire son nom du fleuve Bétis qui la parcourt avant de se jeter dans l'océan :

Betica es noble provinçia en España de las tres mas principales, dicha del rio Beti o

Guadalquivir que entra en el oceano y corre por la Betica ${ }^{71}$.

Cette définition close sur elle-même - la Bétique tire son nom du fleuve Bétis qui traverse la Bétique - laisse un certain flou quant aux contours de la région désignée, dont les limites nord-sud ne sont pas précisées, et laisse en suspens la question de l'appartenance du royaume de Grenade à la province. Le vocabulaire bilingue latincastillan qu'est l'Universal Vocabulario n'a pas vocation, il est vrai, à s'étendre sur des considérations géographiques. Quelques années auparavant, en revanche, Palencia définissait les contours de la Bétique en des termes plus complets, dans un ouvrage consacré à la toponymie de l'Espagne, le Compendiolum de oblitteratis mutatisque nominibus prouinciarum fluminumque Hispaniae ${ }^{72}$. Il y affirmait que la « Province Bétique » allait, d'est en ouest, de la naissance du fleuve Guadalquivir (Bétis) à l'embouchure du fleuve Guadarrama (Anas) et, du nord au sud, de ce même fleuve jusqu'à la mer Mediterranée :

[Provincia Betica], quae ab origine Betis usque in oras Oceani iuxta exitum Anae fluminis in oceanum occidentalem praetenditur et ab ipso Ana usque ad Mediterraneum meridionale dilatatur ${ }^{73}$.

Or, dans cette définition, l'inclusion du royaume de Grenade dans la Bétique est évidente.

Le toponyme Betica, tel que l'emploie Palencia, est donc indiscutablement polyréférentiel, puisqu'il désigne tantôt l'Andalousie chrétienne, tantôt la totalité de l'étendue de la Bétique romaine : étant donnée l'extension de cette dernière vers le sud jusqu'aux rivages de la Méditerranée, elle inclut implicitement, à l'époque où Palencia écrit, le royaume de Grenade. D'ailleurs, les deux acceptions de Betica ne sont nullement contradictoires, dans la mesure où l'auteur, porte-parole de la chrétienté, vise à inclure le royaume de Grenade dans une future Andalousie chrétienne aux limites élargies. Ce n'est pas en vain qu'il rappelle la conquête de Gibraltar par Ferdinand IV et la commente en écrivant : «[...] quidem urbis [...] Fernandus rex Castellae [...] valuit recuperare in maximam totius Beticae amplitudinem $[. . .]^{74}$.» 


\subsection{Vandalia} s'enrichit - encore dans sa relation à un autre toponyme polysémique, présent lui aussi dans le texte de Palencia : Vandalia. Ce toponyme, à l'histoire duquel nous avons consacré un précédent travail ${ }^{75}$, apparaît à plusieurs reprises dans la première Décade. Contrairement à Betica, il ne remonte pas à l'époque romaine ni à l'époque wisigothique. On le trouve pour la première fois, dans l'historiographie chrétienne, dans l'œuvre de Rodrigo Jiménez de Rada (milieu du XIII ${ }^{\mathrm{e}}$ siècle) : d'abord, dans le De Rebus Hispaniae, où il désigne tantôt les terres péninsulaires occupées par les musulmans, tantôt les territoires sous domination musulmane de part et d'autre de la Méditerranée, puis dans la Historia Hugnorum, où il ne désigne plus que le territoire de la péninsule attribué aux VandalesSilinges en 411, à savoir la Bétique ${ }^{76}$.

\section{Vandalia, autre nom de l'Andalousie chrétienne}

$\mathrm{Au}$ début de la première Décade, dans un chapitre consacré au règne de Jean II et à ses relations avec son cousin Henri, infant d'Aragon et maître de Santiago, Palencia écrit : « Henricus [...] in Vandaliam commigravit, cuius provinciae omnes civitates preter Hispalim post dies paucos habuit obsequentes ${ }^{77}$. " Cette province nommée Vandalia, qui inclut Séville et d'autres villes, désigne ici incontestablement l'Andalousie chrétienne. Le toponyme est d'ailleurs employé deux fois dans le même chapitre, en alternance avec Betica. De manière tout aussi explicite, cette équivalence est confirmée dans un chapitre postérieur, où Palencia relate comment Henri IV réunit une armée de trois mille soldats castillans pour que « iuncta cetratis Vandaliae ceu Beticae provinciae Mauros opprimeret ${ }^{78}$ ».

\section{Vandalia, autre nom du royaume de Grenade}

Dans d'autres cas, le référent spatial du toponyme Vandalia est le royaume de Grenade. Ce lien référentiel apparaît clairement lorsque Palencia, en évoquant l'une des premières campagnes d'Henri IV et de ses troupes contre Grenade parle de "latior via adeundae Vandaliae $^{79}$ » (« le chemin le plus commode pour attaquer la Vandalie »). C'est également explicite lorsque l'auteur explique comment le roi reconnaît l'ardeur de l'archevêque de Tolède Alfonso Carrillo à exterminer les infidèles qui « infidelium qui ignominiosa Vandaliae occupatione infamem reddiderint nobilitatemque sceptrique magnitudinem ${ }^{80} »$.

\section{Vandalia, ou la réunion de l'Andalousie et de Grenade}

Si Betica peut renvoyer à deux référents spatiaux, Vandalia, qui renvoie tantôt à l'Andalousie chrétienne tantôt au royaume de Grenade désigne, dans d'autres cas, un troisième ensemble spatial, dont Palencia donne un aperçu dans le Compendiolum. En effet, dans son traité de 1482 sur la toponymie de la Péninsule, il évoque "Vandaliae haud exigua pars a Mauris Granatensibus occupata ${ }^{81}$ " (« une partie importante de la Vandalie occupée par les Maures grenadins »). Vandalia apparaît donc ici comme un ensemble spatial dont le royaume de Grenade ne constitue qu'une partie.

Le puzzle ébauché dans le Compendiolum, continue de se mettre en place dans la première Décade. Ainsi, au début du livre III Palencia évoque le discours dans lequel Henri IV incite 
la noblesse à conquérir "Vandaliae maxima pars quae occupatur a Mauris Granatensibus ${ }^{82}$ " ( la majeure partie de la Vandalie, qui est occupée par les Maures grenadins »). Plus loin, l'auteur décrit la place de Lorca comme la « clé de la Vandalie » (« clavis Vandaliae ») avant de donner une définition de ce dernier toponyme, puis de décrire les contours du royaume de Grenade :

Vandalia quidem omnis ea provincia vocitatur quae a Carthagine Nova usque ad littora Oceani Anam flumen excipientia porrigitur, totamque Beticam in se continet atque Granatae regnum quod ab oriente Lorca, ab occasu autem Oretania vel Tarifa proxima Calpi intercepit 83.

Le royaume de Grenade, qualifié encore de " angulu[s] Vandaliae $e^{84}$ », est bel est bien inclus, dans ces exemples, dans l'entité territoriale Vandalia.

Ainsi, le toponyme Vandalia tel que l'utilise Palencia a un triple référent spatial : lorsqu'il ne renvoie pas au royaume de Grenade, il désigne tantôt l'Andalousie chrétienne (comme Betica, dans une partie de ses emplois), tantôt l'ensemble territorial constitué de l'Andalousie chrétienne et du royaume de Grenade (de même que Betica, dans sa deuxième acception étudiée précédemment).

Le lien référentiel entre les toponymes Betica et Vandalia et les étendues territoriales qu'ils recouvrent est donc d'une grande complexité. Il s'explique pourtant, en premier lieu, par le fait que Palencia, qui a pour modèle la toponymie romaine, est un homme du $\mathrm{xv}^{\mathrm{e}}$ siècle. Son choix du latin comme langue d'expression l'oblige donc à appliquer la toponymie latine à un contexte historico-politique extrêmement différent de celui dans lequel elle s'est constituée ${ }^{85}$. En outre, la confusion apparente entre les deux toponymes ( Betica, Vandalia) et leurs trois référents spatiaux coexistants (l'Andalousie chrétienne, le royaume de Grenade et l'association des deux) reflète la volonté chrétienne d'abolir toute distinction entre les deux États. Elle préfigure l'Andalousie de l'après 1492, qui inclura le royaume de Grenade, même si, par habitude, le nom de ce dernier subsistera longtemps dans les textes. Pour l'heure, comme on vient de le voir, si le royaume de Grenade est délimité par Tarifa à l'ouest et Lorca à l'est, ses limites au nord ne sont pas spécifiées, parce que vouées à être mouvantes.

\section{Géographie de la frontière de Grenade}

Si Palencia peine à délimiter, et donc à désigner les frontières du royaume de Grenade, le petit État musulman bénéficie pourtant, au sein de son discours, d'un privilège que ne lui accordent pas les chroniques en castillan. L'auteur, en effet, dans la veine du courant humaniste auquel il appartient, s'intéresse particulièrement à la géographie, discipline délaissée par les érudits du Moyen Âge et que les Italiens remettent au goût du jour ${ }^{86}$. C'est d'ailleurs en $1482^{87}$, année du début de la guerre de Grenade, qu'Alfonso de Palencia achève son Compendiolum ${ }^{88}$.

\subsection{Des frontières naturelles plutôt que politiques}

Il est vrai que les chroniques en castillan de l'époque des Rois catholiques s'attachent à décrire le milieu physique des villes musulmanes assiégées dans un souci d'intelligibilité de la stratégie militaire chrétienne au sein du récit ${ }^{89}$. Palencia, s'il décrit lui aussi l'environnement orographique particulier de certaines villes, est le seul à donner également une vision géographique d'ensemble du royaume de Grenade. 

dessinent ainsi les contours et les reliefs du royaume de Grenade. Le plus frappant se trouve dans le livre I de Bellum, dans ce bref résumé de la reconquête chrétienne de la péninsule :

Hinc itaque successit in diuturnis seculis regibus multis Castellanorum qui Legionensi primae maiestati suam praeficere antellationem valuerunt lata possessio usque ad montes arduos qui mediterraneo mari supereminent ab oriente in occidentem porrecti videlicet a Carthaginis novae portu quem nostri habent usque ad Calpem, quae civitas fraeto perangusto Europam ab Africa disterminans, Oceano ibidem contigua fuit a nostris forte recuperata Henrici temporibus quarti huius nominis in Hispania Regis parvum animati Granatensium excidio ${ }^{90}$.

Ce passage est particulièrement frappant dans la mesure où, en quelque sorte, il cartographie par les mots le royaume de Grenade, auquel il attribue, à défaut de frontières politiques, des frontières naturelles. Ces dernières sont d'abord constituées par des montagnes escarpées qui, selon l'auteur, ont constitué jusqu'alors la limite des avancées chrétiennes. Dans un passage du livre IX de Bellum, ces reliefs sont également présentés, plus clairement encore, comme des obstacles auxquels se sont heurtés, jusquelà, les successeurs de Pelayo :

[...] granatense regnum, quod Africanae opes asperitasque velut inaccessa locorum in quibus Mauri Granatenses extremam habitationem habuerant tutabantur. Unde illis aucta fuit confidentia perpetuae permansionis [... $]^{11}$.

\subsection{Utilisation idéologique du discours géographique}

Ce type de considération relève, à n'en pas douter, d'un véritable intérêt intellectuel pour la géographie, dont témoigne aussi la rédaction du Compendiolum. Chez Palencia, cependant, rien n'est jamais gratuit, et son intérêt authentique pour la géographie ne l'empêche en rien de la mettre au service de sa pensée politique et, notamment, de sa critique d'Henri IV et de son clan. Dans cette perspective, il n'hésite pas à montrer comment les ambassadeurs d'Henri, envoyés auprès du pape pour obtenir une bulle de croisade, justifient un besoin accru de moyens financiers en mettant en avant - selon un procédé que l'auteur considère comme hypocrite - les reliefs escarpés du royaume de Grenade :

[...] sed tamen debellationem Maurorum difficilem videri, nisi omnes regni copiae cogerentur ut obsidionibus oppugnationibusque necnon tormentis expugnarentur oppida munitissima, quorum situ operibusque confisi Granatenses intra montes alpestres rupesque fere inaccessibiles Vandaliae resideant ${ }^{92}$.

Ailleurs, il utilise lui-même ce thème pour critiquer plus directement Henri IV, accusé par Palencia, comme par beaucoup d'autres, de maurophilie. Ainsi, il lui reproche d'organiser délibérément une expédition vouée à l'échec, en choisissant d'attaquer Grenade par les montagnes plutôt que par les côtes, plus faciles d'accès :

Ceterum ille apparatus bellicus videbatur insipidus ostentationeque fultus quum latior via adeundae Vandaliae neglecta sit atque arctior inutiliorque queratur -maritima enim expreditione opportunius intercepisset Henricus afflixissetque Granatenses, quorum littora patent maritimisque presidiis carent- quam si omissa hac commoditate exercitum ducat per clivos et anfractus propensum infortuniis; [... $]^{93}$.

49 La supposée maurophilie d'Henri, sa négligence à combattre les musulmans, voire sa bienveillance à leur égard, largement illustrées dans la première Décade, constituent l'un des fondements de sa critique virulente du souverain. Mais, si l'œuvre de Palencia est

ILCEA, 18 | 2013 
imprégnée par sa haine envers Henri, elle est également marquée par la virulente maurophobie du chroniqueur, qui, dans la même logique, soutient inconditionnellement Isabelle et Ferdinand dans leur détermination à faire tomber Grenade.

Ainsi, lorsqu'il rapporte la réponse faite par le roi Ferdinand au Sultan égyptien - auprès duquel les Grenadins se sont plaint des agressions castillanes - Palencia reprend à son compte le discours royal qui présente les musulmans comme des occupants effectifs mais illégitimes de terres péninsulaires, contrairement aux chrétiens, qui en sont les propriétaires de droit :

Notissimum esse etiam Soldano caeterisque Mahometistis violenter fraudulenterque Arabes quondam occupasse Hispanias, ut multas per orbem provincias quas Chrystiani patrio jure possidebant. Itaque occupata per injuriam dominia jure a legitimis heredibus recuperari posse $[. . .]^{94}$.

51 Après avoir retracé brièvement la reconquête chrétienne de l'Espagne ${ }^{95}$, Palencia poursuit sa condamnation de la présence de l'islam dans cette partie de la Péninsule, dont les chrétiens sont les propriétaires légitimes ( $j$ just[os] possessor $[e s]^{96} »$ ) et dont il faut expulser les musulmans qui l'occupent par la violence (« expelli a terris violenter occupatis ${ }^{97}$ »). Dans la première Décade, déjà, il rapportait les propos des « adulateurs » d'Henri, qui vantaient les mérites du roi en affirmant que, contrairement à lui, de mauvais chrétiens (rois, nobles et peuple) avaient jusque-là permis aux Maures, « barbares infidèles [...] issus des immondices de l'Afrique", "non seulement de demeurer dans les entrailles de l'Hispanie mais de les ravager ${ }^{98}$. Si les propos virulents rapportés dans ce passage sont critiqués pour leur hypocrisie, l'auteur y adhère manifestement sur le fond.

Palencia reconnaît donc au royaume de Grenade, à défaut de frontières politiques, des frontières physiques. Néanmoins, s'il admet que ces dernières contribuent à la survie du royaume nasride et l'expliquent en partie, il souligne le fait qu'elles ont trop longtemps été utilisées comme excuse au manque de volonté des souverains chrétiens. Si les reliefs, qui constituent pour Grenade une frontière naturelle protectrice, expliquent partiellement la permanence de l'islam dans l'ancienne Bétique, ils ne la justifient aucunement; et la détermination d'Isabelle et deFerdinand à mettre fin à la présence d'un pouvoir politique musulman dans la péninsule finira par avoir raison d'eux, ainsi que du royaume de Grenade tout entier.

\section{Conclusion}

53 Les frontières entre la Castille et les États chrétiens voisins sont des frontières au sens moderne du terme, qui marquent les limites territoriales de l'exercice d'un pouvoir politique reconnu et accepté ; c'est pourquoi elles sont clairement désignées dans le texte de Palencia, comme dans les chroniques en castillan.

Or, le royaume de Grenade n'a jamais été considéré par la Castille comme le siège d'un pouvoir politique définitif : le lien de vassalité entre le roi de Grenade et celui de Castille qui existe dès 1246 et les trêves, par définition transitoires, qui régissent leurs relations, démontrent que la situation de paix, pour la Castille, est considérée dès le départ comme provisoire. En outre, à partir de 1482, l'extension territoriale de l'émirat commence à se réduire au fil des conquêtes chrétiennes. Il est donc logique que les mots de Palencia ne parviennent pas davantage que ceux de ses pairs à donner corps à des limites d'abord niées, puis repoussées et enfin abolies. La complexité de la toponymie palentinienne, qui mêle des référents spatiaux issus de contextes historiques et idéologiques différents, nie 
elle aussi l'existence de la frontière de Grenade. Moins contradictoire qu'il n'y paraît, elle reflète la volonté de refermer la parenthèse musulmane et de renouer avec l'ancienne unité territoriale et religieuse, pour rendre à la chrétienté l'ancienne Bétique dans son extension initiale et, au-delà, tout le territoire de l'Hispanie. Cette unité ne peut être restaurée que par la détermination politique, capable sinon de soulever, du moins de franchir les montagnes escarpées de Grenade, seules frontières auxquelles Palencia reconnaît une existence, lorsqu'il laisse provisoirement le géographe se substituer à l'historien.

\section{BIBLIOGRAPHIE}

\section{Sources}

PALENCIA Alfonso de, Bellum adversus Granatenses, ms. 1627 de la Biblioteca Nacional de Madrid.

-, Compendiolum de oblitteratis mutatisque nominibus prouinciarum fluminumque Hispaniae (Compendio sobre los nombres ya olvidados o mudados de las provincias y ríos de España), 1482, ms. 882 de la bibliothèque de l'abbaye de Montserrat. Disponible sur <www.lluisvives.com/servlet/SirveObras/ abad/57959953116027162922202/index.htm>.

-, Universal Vocabulario en latín y en romançe, Séville, Paulo de Colonia, Magnus Herbst et Thomas Glockner, 1490.

-, Décadas II y III, ms. 2559 de la Biblioteca Universitaria de Salamanca.

-, Cuarta Década, José López de Toro (éd. et trad.), Madrid, Real Academia de la Historia, 1971, 2 t.

-, Guerra de Granada, Antonio Paz y Mélia (éd. facs.), Rafael Peinado Santaella (éd.), Grenade, Universidad de Granada (Archivum, 66), 1998.

-, Gesta Hispaniensia ex annalibus suorum dierum collecta, Brian Tateet Jeremy Lawrance(éds), Madrid, Real Academia de la Historia, 1999, 2 t.

\section{Travaux critiques}

BAZZANA André, «El concepto de frontera en el Mediterráneo Occidental en la Edad Media », Actas del congreso La frontera oriental nazarí como sujeto histórico (s. XIII-XVI), Almería, Instituto de Estudios Almerienses / Diputación de Almería, 1997, p. 25-46.

BURÉSI Pascal, « Nommer, penser les frontières en Espagne aux XI ${ }^{\mathrm{e}}-\mathrm{XIII}{ }^{\mathrm{e}}$ siècles », dans Carlos de Ayala Martínez, Pascal Burési et Philippe Josserand (coord.), Identidad y Representación de la frontera en la España medieval (siglos XI-XIV), Madrid, Casa de Velázquez, Universidad Autónoma de Madrid, « Collection de la Casa de Velázquez, 75 », 2001, p. 51-74.

-, « De la Bétique à l'Andalousie : trace toponymique et fiction étymologique dans l'historiographie médiévale de la péninsule ibérique », Tigre, $\mathrm{n}^{\circ}$ 14, La Trace (2), Trace et histoire, 2006, p. 117-131. 
CARETTE Alice, La représentation de la frontière de Grenade dans les chroniques royales particulières castillanes des XIV et $X V^{e}$ siècles, thèse d'études ibériques, Lyon, ENS-LSH /CIHAM-UMR 5648, 2007, 2 vol., $490 \mathrm{p}$.

CODOÑER Carmen, « El latín en España en época de los Reyes Católicos », Ínsula, nº 691-692, juilletaoût 2004, p. 4-6.

LADERO QUESADA Miguel Ángel, Granada, Historia de un país islámico (1232-1571), $3^{\mathrm{e}}$ éd., Madrid, Gredos, 1989 (1 ${ }^{\text {re }}$ éd. 1969).

-, La Guerra de Granada (1482-1491), Grenade, Diputación de Granada, coll. « Los Libros de la Estrella, 4 », 2001.

-, « Sobre la evolución de las fronteras medievales hispánicas (siglos XI a XIV) », dans Carlos de Ayala Martínez, Pascal Burési et Philippe Josserand (coord.), Identidad y Representación de la frontera en la España medieval (siglos XI-XIV), Madrid, Casa de Velázquez, Universidad Autónoma de Madrid, « Collection de la Casa de Velázquez, 75 », 2001.

Maravall José Antonio, El Concepto de España en la Edad Media, Madrid, Instituto de Estudios Políticos, 1954.

MARTIN Georges, « Toponimia y “avidez de los reyes”: doble lexicalización de los territorios hispanos en la Historia legionensis (llamada silensis) » [en ligne], e-Spania, disponible sur <http://espania.revues.org/21070> (mis en ligne le 11 juin 2012, consulté le 28 août 2012).

ORDUNA Germán, « Movilidad de la frontera castellana y su reflejo en la lengua y la literatura medieval de Castilla », Anales de Historia Antigua y Medieval, 1980-1981, 1982, p. 258-270.

PARDo Madeleine, Alonso de Palencia, historien, études sur les « Gesta Hispaniensia », Lille, Presses du Septentrion, 2002.

SÉNAC Philippe, «'Ad castros de fronteras de mauros qui sunt pro facere'. Note sur le premier testament de Ramire ${ }^{\text {er }}$ d'Aragon ", dans Carlos de Ayala Martínez, Pascal Burési et Philippe Josserand (coord.), Identidad y Representación de la frontera en la España medieval (siglos XI-XIV), Madrid, Casa de Velázquez,Universidad Autónoma de Madrid, « Collection de la Casa de Velázquez, 75 », 2001, p. 205-221.

TATE Robert Brian, « La geografía humanística y los historiadores españoles del siglo XV », Actas del IV Congreso de la AIH, 1971, vol. 2, p. 691-698.

TOUBERT Pierre, «Frontière et frontières : un objet historique ", dans Castrum 4 : Frontière et peuplement dans le monde méditerranéen au Moyen Âge, Rome-Madrid, École française de Rome / Casa de Velázquez, 1992, p. 9-10.

\section{NOTES}

1. Voir à ce sujet les travaux de Martín Ríos Salona, notamment, "La "Reconquista": una aspiración peninsular? Estudio comparativo entre dos tradiciones historiográficas » [en ligne], Bulletin du centre d'études médiévales d'Auxerre (BUCEMA), hors-série $\mathrm{n}^{\circ} 2,2008$, dispoible sur < http://cem.revues.org/index9702.html> (mis en ligne le 24 janvier 2009, consulté le 30 juillet 2012).

2. Voir A. Carette, La représentation de la frontière de Grenade dans les chroniques royales particulières castillanes des XIV et $X V^{e}$ siècles, thèse d'études ibériques, Lyon, ENS-LSH / CIHAM-UMR 5648, 2007, vol. 1, p. 46-79. 
3. Dans une certaine limite, les incursions musulmanes ou chrétiennes de part et d'autre des lignes de défenses ne sont pas réputées remettre en cause les accords de paix.

4. Sur l'histoire du royaume de Grenade, voir notamment M. Á. Ladero Quesada, Granada, Historia de un país islámico (1232-1571), $3^{\mathrm{e}}$ éd., Madrid, Gredos, 1989 (1 $1^{\mathrm{re}}$ éd. 1969).

5. Voir A. Carette, La représentation de la frontière de Grenade..., vol. 1, p. 18-78.

6. M. Á. Ladero Quesada, «Sobre la evolución de las fronteras medievales hispánicas (siglos XI a XIV) ", dans Carlos de Ayala Martínez, Pascal Burési et Philippe Josserand (coord.), Identidad y Representación de la frontera en la España medieval (siglos XI-XIV), Madrid, Casa de Velázquez, Universidad Autónoma de Madrid, «Collection de la Casa de Velázquez, 75 », 2001, p. 5-49.

7. Ibid., p. 5.

8. "La Edad Media, en su época feudal, no conoce el concepto de frontera, dándose en ella el de "marca" [... ]. La "marca", por su propia naturaleza, alude a una zona inestable, imprecisa, sin organizaciónfija, sin que se dé en ella presencia de un poder segura y administrativamente canalizado." (J.A. Maravall Casesnoves, Estado moderno y mentalidad social, Madrid, 1972, p. 121-132, cité par Ladero Quesada, art. cité, p. 6.)

9. J. A. Maravall Casesnoves, El concepto de España en la Edad Media, Madrid, Instituto de Estudios políticos, 1954, p. 149-150.

10. Ladero Quesada fait allusion à l'utilisation parallèle, à partir du XIII ${ }^{\mathrm{e}}$ siècle, de frontera comme nom commun, pour désigner les frontières entre États chrétiens, et de la Frontera comme toponyme pour désigner la région frontalière entre la Castille et Grenade ; cet emploi spécifique se traduit parfois graphiquement par l'emploi de la majuscule pour le toponyme. Voir infra.

11. A. Bazzana, «El concepto de frontera en el Mediterráneo Occidental en la Edad Media », Actas del congreso La frontera oriental nazarí como sujeto histórico (s. XIII-XVI), Almería, Instituto de Estudios Almerienses / Diputación de Almería, 1997, p. 26-27.

12. "[...] con la nueva situación político militar que resulta de la toma de Córdoba (1236), luego de las de Valencia (1238), Jaén (1246) y Sevilla (1248), el concepto de frontera va evolucionando y, desde ahora, ofrece tres características : 畣 Hablar de frontera implica la consciencia clara de la existencia de un límite conocido y respetado; pasado este límite, cada uno sabe que se encuentra en territorio enemigo, en tierras hostiles. 監 Ocurre también una situación nueva, cuando los acontecimientos entre Estados empiezan a ser cotidianos; sin embargo, existen treguas que vienen a disminuir los efectos de los enfrentamientos. 㽞 En fin, se nota una continuidad, a través de los decenios, en los esfuerzos para establecer un límite claro, materializado lo que es nuevo- por una serie de fortificaciones dispuestas según una línea [...]. » (Ibid., p. 42)

13. L'un des premiers à se pencher sur cette question a été G. Orduna, dans son article "Movilidad de la frontera castellana y su reflejo en la lengua y la literatura medieval de Castilla », Anales de Historia Antigua y Medieval, 1980-1981, 1982, p. 258-270.

14. P. Sénac, «'Ad castros de fronteras de mauros qui sunt pro facere'. Note sur le premier testament de Ramire I ${ }^{\text {er }}$ d'Aragon ", dans C. de Ayala Martínez, P. Burési et P. Josserand (coord.), Identidad y Representación de la frontera en la España medieval (siglos XI-XIV), ouvr. cité, p. 211-212.

15. À propos du consensus chrétien qui débouche en 1212 sur la Victoire chrétienne de las Navas de Tolosa, Pascal Burési écrit: "Cet exemple n'est qu'un des jalons qui, du XI ${ }^{\mathrm{e}}$ au XIII ${ }^{\mathrm{e}}$ siècle, conduisent de la domination de l'islam sur une grande partie de la péninsule à sa réduction par étapes, au moins en tant qu'État constitué, au royaume de Grenade. Dans une période de formation territoriale et de "différenciation politique" des monarchies occidentales, il illustre l'émergence d'une "chrétienté hispanique", c'est-à-dire du sentiment, par-delà les conflits, d'une communauté religieuse et culturelle face à l'islam; au même moment se forment au Maghreb et en Andalus les empire almoravide et almohade sur des mouvements de réforme religieuse. Ce processus de définition terrritoriale est évidemment très étroitement lié à la notion de frontière et il n'est guère surprenant de voir surgir dans la documentation, à cette époque, le terme nouveau de frontera. Même si la réalité qu'il exprime n'est pas neuve, puisque le contact entre 
puissances musulmanes et chrétiennes existe dans la péninsule depuis l'intervention araboberbère $\mathrm{au} \mathrm{VIII}^{\mathrm{e}}$ siècle, la nécessité de créer un mot nouveau, utilisé dans la pratique juridique mais aussi dans les chroniques, reflète sans aucun doute un changement dans l'appréhension de cette réalité. » (P. Burési, « Nommer, penser les frontières en Espagne aux XI ${ }^{\mathrm{e}}-\mathrm{XIII}^{\mathrm{e}}$ siècles ", dans C. de Ayala Martínez, P. Burési et P. Josserand (coord.), Identidad y Representación de la frontera en la España medieval (siglos XI-XIV), ouvr. cité, p. 52-53.)

16. Voir A. Carette, La représentation de la frontière de Grenade..., p. 26-45.

17. Ibid., p. 46-59.

18. Ibid., p. 59-117.

19. À partir de la victoire de la coalition chrétienne lors de la bataille du Salado, à l'entrée de Tarifa (1340) sur l'alliance musulmane entre Grenadins et Mérinides (qui menaçait d'envahir de nouveau la péninsule), Grenade est définitivement isolée militairement. Dès lors, à l'exclusion de quelques razzias menées par les Maures grenadins en terres chrétiennes, le petit royaume, que la monarchie castillane prétend conquérir, est à la fois l'objet et le théâtre des combats.

20. Ainsi, ir a la frontera est synonyme de ir a la guerra de los moros. Voir A. Carette, La représentation de la frontière de Grenade..., p. 69-74.

21. Sur l'œuvre historiographique de Palencia, voir M. Pardo, Alonso de Palencia, historien, études sur les « Gesta Hispaniensia », Lille, Presses du Septentrion, 2002.

22. Depuis le règne d'Alphonse $X$ le Sage, le Castillan a remplacé le latin comme langue officielle des chroniques.

23. Voir C. Codoñer, «El latín en España en época de los Reyes Católicos », Ínsula, n 691-692, juillet-août 2004, p. 5.

24. Voir A. Carette, La représentation de la frontière de Grenade..., vol. 1, p. 32-35.

25. Dans le livre IV, chapitre I de la première Décade, Palencia évoque la venue en Andalousie de la reine Jeanne, deuxième épouse d'Henri IV, « ex finibus Portugaliae usque Cordubam et postea usque Hispalim [...]» (« de la frontière du Portugal jusqu'à Cordoue puis jusqu'à Séville») (Gesta Hispaniensia ex annalibus suorum dierum collecta, Gesta Hispaniensia ex annalibus suorum dierum collecta, B. Tateet J. Lawrance(éds), Madrid, Real Academia de la Historia, 1999, vol. I, p. 138. [Dorénavant noté $G H]$ ).

26. GH, II, p. 357. Tate et Lawrance utilisent d'ailleurs deux termes différents dans leur traduction de ce passage, respectivement frontera et marcha. On trouve également le substantif finitima suivi du nom latin de la Navarre (« finitima Cantabriae », GH, II, p. 234).

27. GH, I, p. 59.

28. Ibid., p. 63.

29. Ibid., p. 4.

30. Voir par exemple : «[...] proxima Castellae Cantabria incolitur» (GH, I, p. 59); «[...] provincia Legionensi, quae Lusitanie contigua est [...]» (GH, II, p. 359) ; « [...] festina expeditione profiscitur in loca finitima Cantabriae [...]» (GH, I, p. 54).

31. GH, II, p. 352.

32. GH, I, p. 182.

33. Bellum adversus Granatenses, ms. 1627 BNE, fol. 90v. Dorénavant noté Bellum (« avant que le roi Ferdinand ne conduisît son armée sur la frontière des Grenadins »). Voir aussi «[...] dum fines Granatensium remotissimos regia maiestas adiret » («[...] tandis que sa royale majesté se rendait sur la frontière des Grenadins ») (ibid., fol. 77r).

34. Bellum, fol. $24 \mathrm{v}$.

35. L'expression la frontera de (los) Moros alterne, dans les chroniques castillanes, avec le simple syntagme la frontera, lorsque le texte souhaite insister sur la dimension militaire et religieuse du conflit. Voir A. Carette, La représentation de la frontière de Grenade..., vol. 1, p. 55-59.

36. «Vascus de Meyra, [...] qui deceptus a Mauris Granatensibus frumentum vendidit hostibus pro caro ementibus precio; [...]» («Vasco de Meira [...] qui, trahi par les Maures Grenadins, vendit du blé 
aux ennemis à un prix élevé ») (GH, II, p. 241) ; « [...] regnante Iohanne Secundo, quo tempore Mauri Granatenses [...] multas clades in illa provincia Carthaginensi provincialibus intulere [...]» («sous le règne de Jean II, les Maures Grenadins, causèrent de nombreux dommages aux habitants de cette province Carthaginoise ») (GH, II, p. 352).

37. GH, II, p. 287. Voir aussi : «Alhamam [...] quia finitima Granatae » (Bellum, fol. 10v).

38. Lorsque Palencia utilise l'expression « inire Granata » (GH, II, p. 353) pour relater les fréquents déplacements d'Alfonso Fajardo, on ne peut déterminer clairement s'il s'agit de la ville ou du royaume.

39. GH, II, p. 351. Voir infra.

40. «[...] campum granatensem » (GH, I, p. 109); « in campo granatensi » (ibid., p. 111); « in campum granatensem » (ibid., p. 175) ; « in campos Granatensium » (ibid., p. 183) : «per convalles anfractusque Granatensium reducere agmen versus Cordobensium fines » (ibid., p. 113).

41. GH, I, p. 176.

42. Bellum, fol. 81r.

43. Ibid., fol. 28r, 43r, 45r.

44. Ibid., fol. 89v.

45. «[...] in granatensem provinciam» (Bellum, fol. 28r); " [...] in granatensi provincia» (ibid., fol. $94 \mathrm{r}$ ) ; « in remotissimam Granatensium provinciam » (ibid., fol. 47r).

46. " granatensis ditionis» (Bellum, fol. 18v) ; «ditionis granatensis» (ibid., fol. 10r) ; " granatensis ditonis » (ibid., fol. 31r) ; « Abdera et in aliis granatensis ditionis » (ibid., fol. 98r).

47. Le mot est ainsi défini dans l'Universal Vocabulario : «Dicio es poderío, condiçión, mandamiento » ( Universal Vocabulario en latín y en romançe, Sévilla, Paulo de Colonia, Magnus Herbst et Thomas Glockner, 1490, t. 1, fol. 10v. [Dorénavant noté UVoc]). Ditio est employé également pour désigner les territoires de la Castille (GH, I, p. 4). (Voir supra, note 19.)

48. « [...] in regno Granatae [...]» (Bellum, fol. 31r) ; « [...] regni granatensis [...]» (ibid., fol. 97r) ; « [...] maiore regni granatensis partem [...]» (ibid., fol. 135v) ; « [...] granatense regnum [...]» (ibid., fol. 137v). 49. «[...] regibus Granatae» (Bellum, fol. 30r) ; «Rex Granatae Boaudelis» (ibid., fol. 66v) ; «[...] Granatae regem Abohardiglem» (ibid., fol.69v); «[...] rex Granatae Boaudelis junior [...]» (ibid., fol. 118r) ; « Mahometi Granatae regem [...]» (ibid., fol. 119r) ; « [...] Audelis, altrem granatensium rex [...]» (ibid., fol. 120v) ; « [...] Boaudelis Rex Granatae [...]» (ibid., fol. 130v).

50. Voir M. Á. Ladero Quesada, Granada, Historia de un país islámico, ouvr. cité, p. 247-287.

51. Voir A. Bazzana, «El concepto de frontera... », art. cité, p. 27.

52. GH, I, p. 111. Voir aussi «[...] Granatenses ductu Alfonsi Faiardi [...] nocentissimis incursionubus fines nostros populabantur [...]» (« les Grenadins, conduits par Alfonso Fajardo [...], dévastaient nos frontières par leurs très néfastes incursions [...]») (GH, II, p. 229).

53. GH, I, p. 145.

54. GH, II, p. 313 : «Fajardo, qui possédait Carthagène et tous les lieux frontaliers des Maures et proches des rivages de la Méditerrannée.»

55. Ibid., p. 287.

56. GH, I, p. 147 : « il ordonna également de renforcer le reste des frontières entre l'Andalousie et les Grenadins ainsi que les forteresses qui en étaient les plus proches ». Voir aussi «[...] praesidium christianae militiae Mauris opposita [...] » (« la forteresse de l'armée chrétienne qui faisait face aux Maures ») (Bellum, fol. 107v).

57. Ibid., p. 113.

58. On trouve par exemple le complément de lieu "in beticos fines", dans le livre 28 de la troisième Décade, ms. 2559 de la Biblioteca Universitaria de Salamanca (dorénavant BUSal), fol. 338v.

59. Voir note ci-dessus. Voir aussi Bellum, fol. 39r. 
60. Voir à ce sujet A. Carette, «De la Bétique à l'Andalousie : trace toponymique et fiction étymologique dans l'historiographie médiévale de la péninsule ibérique », Tigre, $\mathrm{n}^{\circ} 14$, La Trace (2) , Trace et histoire, 2006, p. 117-131.

61. Ibid., p. 124.

62. Voir infra, note 85.

63. Voir A. Carette, « De la Bétique à l'Andalousie... », art. cité.

64. GH, I, p. 146 : «Parallèlement, la cour se dirigea, à travers la Bétique, de Jaén à Cordoue, de Cordoue à Ecija, de là à Carmona et enfin à Séville [...].

65. GH, II, p. 250 : «Tandis que le roi parcourait la Bétique, il était sollicité chaque jour par des envoyés du marquis [...]. » Voir aussi, parmi de nombreux exemples : «[...] in Beticam se conferat situmque Malace contemplatur», (GH, I, p. 157) ; "[...] ne elati Granatenses consciique confusionis nostrae Betica devastarent » (GH, II, p. 238) ; « [...] totius Beticae [...]»] (GH, II, p. 240) ; « [...] ex Betica nihil opis futurum habebatur compertum » (GH, II, p. 418).

66. Ms. 2959 BUSal, fol. 72r. Voir aussi : « in provinciae Betica » (GH, I, p. 14) ; « ex Betica provincia » ( GH, I, p. 17). Voir aussi : "Et secum in provinciam Beticam duxit almirantum Federicum [...] " (" et il emmena avec lui dans la province Bétique l'amiral Fadrique [...]») (ibid., p. 107); «Rex quidem Granatae [...] ingentes copias coegit ut repente posset populari haud exiguam Beticae provinciae partem [... ]» («Le roi de Grenade rassembla de nombreuses troupes afin de pouvoir dévaster rapidement une grande partie de la province Bétique [...] ») (GH, II, p. 237).

67. Voir GH, I, p. 77.

68. GH, I, p. 188, Bellum, fol. 21r, GH, II, p. 237, respectivement.

69. GH, I, p. 109.

70. GH, II, p. 309.

71. Voir UVoc, fol. 45r.

72. A. de Palencia, Compendiolum de oblitteratis mutatisque nominibus prouinciarum fluminumque Hispaniae (Compendio sobre los nombres ya olvidados o mudados de las provincias y ríos de España), 1482, ms. 882 de la bibliothèque de l'abbaye de Montserrat.

73. Compendiolum, fol. $25 \mathrm{v}$.

74. GH, II, p. 240. En effet, si le syntagme " in maximam amplitudinem Beticae " peut être pris au sens figuré (apportant ainsi une grandeur supérieure à l'Andalousie [chrétienne]), il est tout aussi significatif, sinon plus, au sens propre (donnant ainsi une plus grande extension à l'ensemble de l'Andalousie).

75. Voir supra, note 51.

76. Voir A. Carette, « De la Bétique à l'Andalousie... », art. cité, p. 124-128.

77. GH, I, p. 13.

78. Ibid., p. 104 : «afin que, jointe aux soldats de la Bétique ou Vandalie, elle anéantît les Maures ".

79. Ibid., p. 108.

80. Ibid., p. 157 : «par leur occupation ignominieuse de la Vandalie, avaient jeté l'infamie sur la noblesse et la grandeur de la couronne ».

81. Compendiolum, fol. $25 \mathrm{v}$.

82. GH, I, p. 96.

83. GH, II, p. 351 : «Est appelée Vandalie toute cette province qui s'étend depuis Carthagène jusqu'aux rivages de l'Océan, où débouche le fleuve Anas (Guadiana), et contient en elle toute la Bétique ainsi que le royaume de Grenade, lequel est interrompu à l'est par Lorca et à l'ouest par Oretania ou Tarifa. »

84. GH, I, p. 107.

85. Le cas de Palencia a des précédents dans l'historiographie latine de la Péninsule. Voir à ce sujet le récent article de G. Martin, «Toponimia y “avidez de los reyes”: doble lexicalización de 
los territorios hispanos en la Historia legionensis (llamada silensis) » [en ligne], e-Spania, disponible sur <http://e-spania.revues.org/21070> (mis en ligne le 11 juin 2012, consulté le 28 août 2012).

86. Voir R. B. Tate, «La geografía humanística y los historiadores españoles del siglo XV », Actas del IV Congreso de la AIH, 1971, vol. 2, p. 691-698.

87. Compendiolum, fol. $27 \mathrm{v}$.

88. Voir supra, note 63.

89. Voir A. Carette, La représentation de la frontière de Grenade..., vol. 1, p. 139-150.

90. Bellum, fol. $1 \mathrm{v}$ : "C'est pourquoi, il advint que, au cours de longs siècles, certains rois des Castillans, qui s'imposèrent sur les premiers rois de Léon, étendirent leurs conquêtes jusqu'aux montagnes escarpées qui, de l'orient à l'occident, se dressent face à la Méditerranée, c'est-à-dire depuis Carthagène, en possession des nôtres, jusqu'à Gibraltar : cette ville, séparée de l'Afrique par un étroit bras de mer et bordée par l'Océan, fut reprise fortuitement par les nôtres du temps d'Henri, quatrième roi de ce nom en Espagne peu enclin à l'extermination des Grenadins [...]. »

91. Bellum, fol. $137 \mathrm{r}$ : «[...] le royaume de Grenade, que protégeaient les aides venues d'Afrique ainsi que le caractère inaccessible des lieux, dans lesquels les Maures grenadins avaient trouvé leur ultime refuge. C'est pourquoi leur espoir d'y demeurer perpétuellement s'accrut. »

92. GH, I, p. 144 : « Il semblait cependant difficile de vaincre les Maures sans obliger toutes les forces du royaume à attaquer, par des sièges, des assauts et en utilisant des catapultes ces villes fortes, parmi les montagnes escarpées et les roches reculées de la Vandalie, où vivent les Grenadins, confiants dans leurs positions et leurs défenses. »

93. ibid., p. 108: "D'autre part, cet appareil guerrier semblait être une ostentation vaine et apparente, dans la mesure où l'on avait négligé la voie la plus commode pour envahir la Vandalie et recherché la plus étroite et inutile - avec une expédition maritime, en effet, Henri aurait assiégé et affligé davantage les Grenadins, dont les côtes sont ouvertes et dépourvues de défenses maritimes - et, au lieu de profiter de cette commodité, il conduisit son armée à travers les pentes et les gorges, l'exposant aux revers. "

94. Bellum, fol.137r : «Il était notoire pour le Sultan et les autres Mahométans que les Arabes avaient autrefois occupé les Espagnes avec violence et perfidie, de même que de nombreuses autres provinces à travers le monde dont les chrétiens étaient les propriétaires légitimes. C'est pourquoi des territoires occupés injustement pouvaient être repris par leurs héritiers légitimes. "

95. Voir supra, note 83.

96. Bellum, fol. 137v.

97. Ibid.

98. «[...] in visceribus Hispaniae [...] non tantum permanere sed grassari [...] atque possideri [...] optimam partem Hispaniae ab inermi paucitate infidelium barbarorum [...] ex colluvie Libica [...]» («[Ils] avaient non seulement permis aux Maures de demeurer dans les entrailles de l'Espagne, mais aussi de les ravager et laissé la meilleure partie de l'Espagne être possédée par une poignée de Barbares infidèles issus des immondices de l'Afrique ») (GH, I, p. 103).

\section{RÉSUMÉS}

Ce travail interroge les expressions et la perception de la frontière de Grenade dans les Gesta Hispaniensia et Bellum adversus Granatenses d'Alfonso de Palencia (1423-1492). Il montre d'abord 
comment, par ses choix lexicaux et syntaxiques, l'auteur - dont la langue d'expression est le latin humaniste - réserve à la frontière avec le royaume de Grenade un traitement distinct de celui des autres frontières de la Castille. Il explique ensuite comment le caractère polyréférentiel des toponymes Betica et Vandalia reflète, chez l'auteur, une volonté de voir disparaître le royaume de Grenade et renaître l'Hispanie chrétienne unifiée des Wisigoths. Enfin, il démontre comment Palencia, se livrant à des considérations géographiques, reconnaît au royaume de Grenade des frontières naturelles tout en s'appuyant sur la géographie pour prôner la disparition du dernier État musulman d'Espagne.

This article examines the expressions and perception of the frontier of Granada in the Gesta Hispaniensia and Bellum adversus Granatenses by Alfonso de Palencia (1423-1492). Firslty, it shows how, the author, who writes in Humanist Latin, makes use of his lexical and syntaxic options, to treat the frontier with Granada differently than the frontiers between Castile and the others states. Secondly, it explains how the polyrenferential nature of both toponymes Betica and Vandalia reflects the author's desire to see the kingdom of Granada disappear with the rebirth of the ancient unified Wisigothic Hispania. Finally, it proves Palencia is interested in geographic details, assigning natural frontiers to the kingdom of Granada, and also relies on geography when he advocates bringing to an end the last Muslim State of Spain.

Este trabajo cuestiona las expresiones y la percepción de la frontera de Granada en los Gesta Hispaniensia y Bellum adversus Granatenses de Alfonso de Palencia (1423-1492). Muestra primero cómo, mediante sus elecciones léxicas y sintácticas, el autor -cuya lengua de expresión es el latín humanístico- plasma la frontera con el reino de Granada de manera distinta de las demás fronteras de Castilla. Explica, a continuación, cómo el carácter polireferencial de los topónimos Betica y Vandalia reflejan, por parte del autor, una voluntad de que desaparezca el reino de Granada y renazca la Hispania cristiana unificada de los Visigodos. Por fin, demuestra cómo Palencia, al formular observaciones geográficas, le reconoce al reino de Granada unas fronteras naturales, sin dejar de apoyarse en la geografía para abogar por la desaparición del último Estado musulmán de España.

\section{INDEX}

Mots-clés : Alfonso de Palencia, frontière de Grenade, toponymie, géographie

Palabras claves : Alfonso de Palencia, frontera de Granada, toponimia, geografía

Keywords : Alfonso de Palencia, frontier of Granada, toponymy, geography

\section{AUTEUR}

\section{ALICE CARETTE}

Université Grenoble Alpes, ILCEA 